\title{
HIGH TIME RESOLUTION SPECTROSCOPY OF SHORT PERIOD VARIABLE OBJECTS
}

\author{
R. Michel ${ }^{1}$ and J. L. A. Fordham ${ }^{2}$
}

Photon counting detector technology allows high time resolution spectroscopy on sources such as pulsars and cataclysmic variables. Here we report on first observing trials on Cvs in a high time resolution mode undertaken with the MIC photon counting detector (Fordham et al. 2000) on the 2.1m telescope at San Pedro Martir Observatory.

The temporal resolution utilized was $1.8 \mathrm{~ms}$ and data were acquired primarily on UX UMa and V405 Aur. Time resolved spectroscopic images were produced based on the orbital and spin periods for V405 Aur and based on the orbital period for UX UMa. Figure 1 shows the continuum subtracted spectroscopic image of UX UMa based upon its $4 \mathrm{~h} 43 \mathrm{~min}$ orbital period. A number of features can be seen such as:

a) the increase in intensity for the $\mathrm{H}$ lines during eclipse. $\mathrm{H} \beta$ has a change in emission intensity whereas lines such as $\mathrm{H} \delta$ and $\mathrm{H} \varepsilon$ change from absorption to emission,

b) change from absorption to emission for $\mathrm{MgII}$ and HeI (4922) during eclipse,

c) HeII being constant over the period,

d) apparent rotation in the $\mathrm{MgII}$ and $\mathrm{HeI}$ lines during eclipse, and

e) the change in continuum profile related to eclipse. As a normalized mean continuum was used for subtraction, the difference during eclipse becomes apparent.

Figure 2 shows the 545s spin period light curve for V405 Aur derived be collapsing its spin period spectroscopic image in the dispersion dimension. Spectroscopic imaging in this mode allows analysis of spectral changes as a function of phase position, as highlighted in Figure 1, and light curve variation as a function of wavelength as demonstrated by Figure 2 .

\section{REFERENCES}

Fordham J. L. A., Kawakami H., Michel R., Much R., \& Robinson j. R. 2000, MNRAS, 319, 414

\footnotetext{
${ }^{1}$ Instituto de Astronomía, UNAM, Ensenada, México. UK.
}

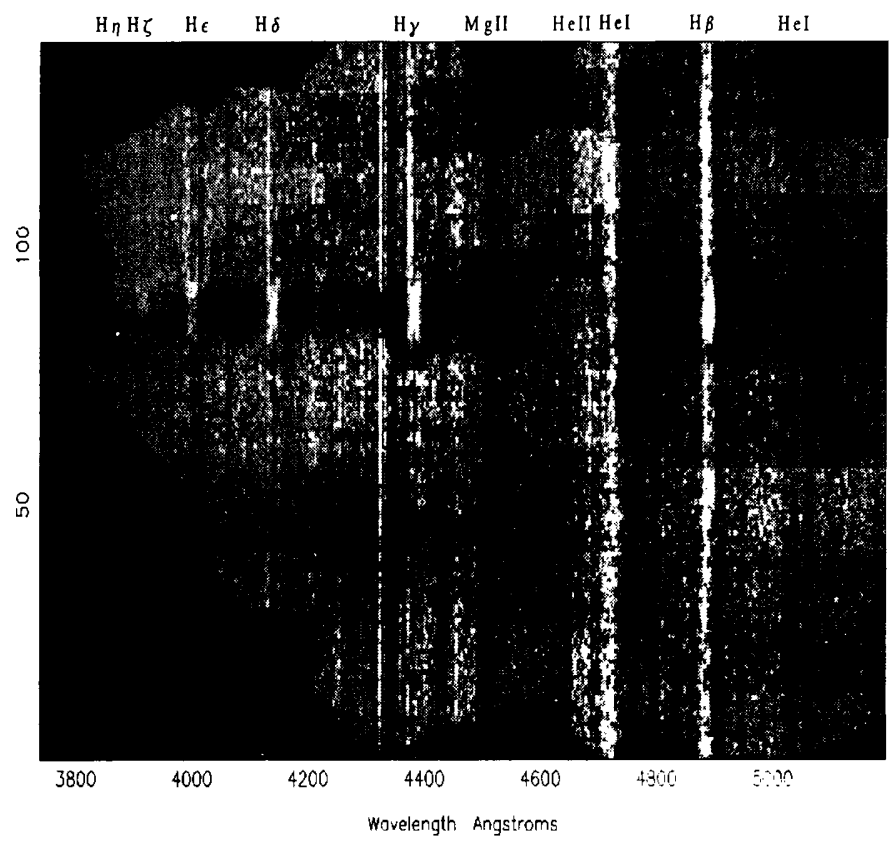

Fig. 1. Time resolved spectral image for UX LNa based upon the orbital period. The image is continuum subtracted to highlight variations in spectral features as a function of phase position. For this image, the orbital period has been split into 140100 s time slices.

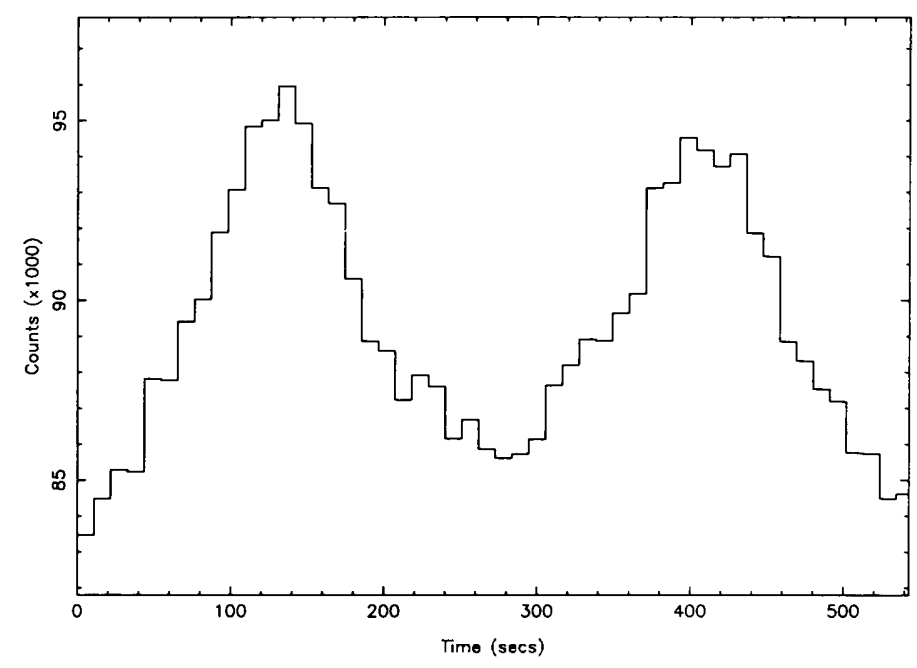

Fig. 2. Spin period light curve of V405 tur showing clearly two peaks per period relating to emission from both poles of the white dwarf. Data wass acquired in February 3rd 2001 with a total integration time of 4.4 hours. The spectra are split into 50 contiguous time slices but temporal resolution can be chosen during data reduction in order to suit signal to noise requirements. 